\title{
THE ROOTS OF A POLYNOMIAL AND ITS DERIVATIVE ${ }^{1}$
}

\author{
JOHN C. MONTGOMERY
}

Consider a polynomial $f(z)$ of degree $n$, with the roots $\alpha_{1}, \cdots, \alpha_{n}$ and its first derivative $f^{\prime}(z)$ with roots $\beta_{1}, \cdots, \beta_{n-1}$. The classical problem, where certain of the roots $\alpha_{i}$ are given and conclusions are drawn concerning the location of the roots $\beta_{i}$, will be considered in the converse. By means of an identity between these two sets of roots, two theorems will be given which restrict the location of the roots of the polynomial $f(z)$ when certain of the roots of its derivative, $f^{\prime}(z)$ are equally placed on the unit circle.

THEOREM 1. If $\alpha_{1}, \cdots, \alpha_{n}$, the $n$ roots of the polynomial $f(z)$ of degree $n$, are different from the $q$ distinct roots $\beta_{1}, \cdots, \beta_{q}, q \leqq n-1$, of the derived polynomial $f^{\prime}(z)$, then

$$
\sum_{j=1}^{n} \frac{1}{\left(\beta_{1}-\alpha_{j}\right)\left(\beta_{2}-\alpha_{j}\right) \cdots\left(\beta_{q}-\alpha_{j}\right)}=0 .
$$

The expansion of $1 /\left(\beta_{1}-x\right) \cdots\left(\beta_{q}-x\right)$ into partial fractions when summed as $x$ ranges over the values $\alpha_{1}, \cdots, \alpha_{n}$, yields

$$
\sum_{x} \frac{1}{\left(\beta_{1}-x\right) \cdots\left(\beta_{q}-x\right)}=\sum_{x} \frac{A_{1}}{\left(\beta_{1}-x\right)}+\cdots+\sum_{x} \frac{A_{q}}{\left(\beta_{q}-x\right)},
$$

where the coefficients $A_{1}, \cdots, A_{q}$ are expressions of $\beta_{1}, \cdots, \beta_{q}$, and thus may be taken outside of the summations. Then the theorem follows since the remaining sums are all identically zero by the well known expression

$$
\frac{f^{\prime}\left(\beta_{i}\right)}{f\left(\beta_{i}\right)}=\sum_{j=1}^{n} \frac{1}{\left(\beta_{i}-\alpha_{j}\right)}=0, \quad i=1,2, \cdots, q .
$$

In a recent paper by M. Marden ${ }^{2}$ an identity is given between $q$ roots of $f^{\prime}(z)$ and $p$ roots of $f(z)$ of degree $n, n=p+q-1$. This identity gave a clue to the relation (1). In fact (1) may be derived by means of a complete induction operating on the Marden identity.

In order to study the effect that (1) has on the distribution of the roots of the polynomial $f(z)$, we introduce the following notation. Let

${ }^{1}$ Taken from a dissertation presented for the degree of Doctor of Philosophy in Yale University.

${ }^{2}$ M. Marden, Kakeya's problem, Transactions of this Society, vol. 45 (1939), pp. $355-368$. 
$g_{j}$ be an arbitrary axis through $\beta_{j}$ making an angle of $\psi$ with the axis of reals. Let $L_{j}^{(k)}$ be the line through $\alpha_{k}$ and $\beta_{j}$ of length $r_{j}^{(k)}$. Let $\theta_{j}^{(k)}$ be the angle formed by $g_{j}$ and $L_{j}^{(k)}$ measured in the positive direction from $L_{j}^{(k)}$ to $g_{j}$. Finally set $\alpha_{k}-\beta_{j}=r_{j}^{(k)} e^{\left(\theta_{i}^{(k)}+\psi\right) t}, j=1,2, \cdots, q$; $k=1,2, \cdots, n$. With these notations the identity (1) may be stated in terms of its real and imaginary parts in the forms

$$
\begin{aligned}
& \sum_{k=1}^{n} \frac{\cos \left(\theta_{1}^{(k)}+\cdots+\theta_{q}^{(k)}\right)}{r_{1}^{(k)} \cdots r_{q}^{(k)}}=0, \\
& \sum_{k=1}^{n} \frac{\sin \left(\theta_{1}^{(k)}+\cdots+\theta_{q}^{(k)}\right)}{r_{1}^{(k)} \cdots r_{q}^{(k)}}=0 .
\end{aligned}
$$

Now in particular let us suppose that the $q$ distinct roots of the polynomial $f^{\prime}(z)$ are on the unit circle at the points $\theta=2 j \pi / q$, $j=1,2, \cdots, q$, respectively. Then if $\psi$ is a right angle, $L_{j}^{(k)}$, for all $j$, will have the equation

$$
\rho \sin \theta-\sin 2 j \pi / q=\cot \theta_{j}^{(k)}(\rho \cos \theta-\cos 2 j \pi / q) .
$$

Since the lines $L_{j}^{(k)}$, for all $j$, intersect at $\alpha_{k}$, a root of $f(z)$, we determine the location of $\alpha_{k}$ so that (2) or (3) will be satisfied, by requiring the $\cos \left(\theta_{1}^{(k)}+\cdots+\theta_{q}^{(k)}\right)=0$ or the $\sin \left(\theta_{1}^{(k)}+\cdots+\theta_{q}^{(k)}\right)=0$. The curve $D$, where the $\cos \left(\theta_{1}^{(k)}+\cdots+\theta_{q}^{(k)}\right)=0$, may be determined by the substitution of $L_{j}^{(k)}$ in the expression for the $\cot \left(\theta_{1}^{(k)}+\cdots+\theta_{q}^{(k)}\right)$ $=0$, and the curve $C$, where the $\sin \left(\theta_{1}^{(k)}+\cdots+\theta_{a}^{(k)}\right)=0$, may be determined by the substitution of $L_{j}^{(k)}$ in the expression $\left[\cot \left(\theta_{1}^{(k)}+\cdots+\theta_{a}^{(k)}\right)\right]^{-1}=0$. We determine the curves $D$ and $C$ if $q$ is even.

Using the following expansions of the cotangent function and dropping the superscript $k$, we have $D$ and $C$ from

$$
\begin{aligned}
0 & =\cot \left(\theta_{1}+\cdots+\theta_{q}\right) \\
& =S_{q}^{q} \cot \theta_{j}-S_{q-2}^{q} \cot \theta_{j}+S_{q-4}^{q} \cot \theta_{j}+\cdots+(-1)^{q / 2}
\end{aligned}
$$

and from

$$
\begin{aligned}
0= & {\left[\cot \left(\theta_{1}+\cdots+\theta_{q}\right)\right]^{-1} } \\
= & S_{q-1}^{q} \cot \theta_{j}-S_{q-3}^{q} \cot \theta_{j}+S_{q-5}^{q} \cot \theta_{j}-\cdots \\
& +(-1)^{q / 2+1} S_{1}^{q} \cot \theta_{j},
\end{aligned}
$$

respectively, where 


$$
\cot \theta_{j}=\frac{\rho \sin \theta-\sin 2 j \pi / q}{\rho \cos \theta-\cos 2 j \pi / q}, \quad j=1,2, \cdots, q,
$$

and $S_{r}^{q} \cot \theta_{i}$ means the sum of all different products that can be formed using the $q$ functions $\cot \theta_{1}, \cdots, \cot \theta_{q}, r$ at a time. We also note that the $\cot \left(\theta_{1}+\cdots+\theta_{q}\right)$ equals the right side of equation (4) divided by the right side of equation (5). Thence the expressions for $D$ and $C$ will follow from the two lemmas.

LeMma 1. If $F(\rho, \theta)=\rho^{q} \cos q \theta+\sum_{k=1}^{q-1} a_{k} \rho^{k} \cos k \theta+b_{k} \rho^{k} \sin k \theta=K$, and if $F(\rho, \theta)$ is identically equal to $F\left(\rho, \theta^{\prime}\right)$ where $\theta^{\prime}=2 \pi / q+\theta$, then $a_{k}=b_{k}=0, k=1,2, \cdots, q-1$.

We compare the terms of like powers of $\rho$ -

$$
\rho^{q}: \quad \cos q \theta^{\prime}=\cos q(2 \pi / q+\theta)=\cos (2 \pi+q \theta)=\cos q \theta,
$$

$\rho^{k}, 0<k<q: \quad a_{k} \cos k \theta^{\prime}+b_{k} \sin k \theta^{\prime}=a_{k} \cos k \theta+b_{k} \sin k \theta$,

or by expressing $\theta^{\prime}$ in terms of $\theta$ and observing that the relation must hold for all values of $\theta$, we have

$$
\begin{aligned}
a_{k}(\cos 2 \pi k / q-1)+b_{k}(\sin 2 \pi k / q) & =0, \\
-a_{k}(\sin 2 \pi k / q)+b_{k}(\cos 2 \pi k / q-1) & =0 .
\end{aligned}
$$

For a solution of $a_{k}$ and $b_{k}$ which is not zero to exist, we get from the determinant of the coefficients that the $\cos 2 \pi k / q=1$ or $2 \pi k / q=2 \pi t$, $t=0,1, \cdots$. Hence $k / q=t$, which is impossible. Therefore the only solution is $a_{k}=b_{k}=0$, for $k=1,2, \cdots, q-1$.

LeMmA 2. If $F(\rho, \theta)=\rho^{q} \sin q \theta+\sum_{k=1}^{q-1} a_{k} \rho^{k} \cos k \theta+b_{k} \rho^{k} \sin k \theta=L$ and $F(\rho, \theta)$ is identically equal to $F\left(\rho, \theta^{\prime}\right)$ where $\theta^{\prime}=2 \pi / q+\theta$, then $a_{k}=b_{k}=0, k=1,2, \cdots, q-1$.

The method of proof is obvious from Lemma 1. Since (4) satisfies the hypothesis of Lemma 1 , we have $D$ as $\rho^{q} \cos q \theta=K$. We also observe that the point $\rho=1, \theta=2 \pi / q$ is on the curve $D$, from which we see that $K=1$. Similarly (5) satisfies the hypothesis of Lemma 2 and thus $C$ is $\rho^{q} \sin q \theta=L$. Also $\rho=1, \theta=2 \pi / q$ is a point on $C$ from which follows $L=0$. Combining the above facts the following theorems may be stated.

THEOREM 2. If $q$ distinct roots of the derivative of the polynomial $f(z)$, of degree $n$, are different from the roots of $f(z)$ and are located so that they divide the unit circle into $q$ equal arcs, then either all of the roots of $f(z)$ are on the curve $\rho^{a} \cos q \theta=1$, or there are roots in the interior as well as the exterior of the curve. 
By the interior is meant all points of the plane which are not on the curve but which are separated from the point $\rho=0$ by the curve. By the exterior is meant all other points of the plane not on the curve nor in the interior of the curve.

THEOREM 3. If, with the hypothesis of Theorem 2 , the $2 q$ radial lines through $\rho=0$ and the roots of $f^{\prime}(z)$ and midway between the roots are drawn so that the plane is divided into $2 q$ sectors, numbered from 1 to $2 q$, then either all the roots of $f(z)$ must lie on the radial lines or there must be roots in an odd as well as in an even numbered sector.

Covina, CALIF.

\section{ON THE REPRESENTATIONS, $N_{7}\left(m^{2}\right)^{1}$}

\section{D. OLDS}

1. Introduction. Write $N_{r}(n)$ for the number of representations of the positive integer $n$ as the sum of $r$ squares, and write $N_{r}(n, k)$ for the number of representations of $n$ as the sum of $r$ squares in which the first $k$ squares in each representation are odd with positive roots, while the remaining $r-k$ squares are even with roots positive, negative, or zero. In a previous paper the author $[5]^{2}$ gave an arithmetical derivation of the formula for $N_{3}\left(n^{2}\right)$. The method used to prove this result was based upon that employed by Hurwitz [2] in his discussion of the analogous formula for $N_{5}\left(n^{2}\right)$.

In 1930, G. Pall [6] gave an analytical derivation of the formula for $N_{7}\left(c n^{2}\right), c$ an integer. His formula shows, in particular, that if $m=p_{1}^{\alpha_{1}} p_{2}^{\alpha_{2}} \cdots p_{s}^{\alpha_{s}}$, where $p_{1}, p_{2}, \cdots, p_{s}$ are distinct odd primes, then

$$
N_{7}\left(m^{2}\right)=14 \prod_{\nu=1}^{s}\left[p_{\nu}, \alpha_{\nu}\right]
$$

where

$$
\left[p_{\nu}, \alpha_{\nu}\right]=\sigma_{5}\left(p_{\nu}^{\alpha_{\nu}}\right)-(-1)^{\left(p_{\nu}-1\right) / 2}{ }_{p_{\nu}}^{2} \sigma_{5}\left(p_{\nu}^{\alpha_{\nu}-1}\right) .
$$

We define the arithmetical function $\sigma_{k}(n)$, which occurs here, and the function $\rho_{k}(n)$, which occurs later, by the sums

${ }_{1}^{1}$ This is the second part of a paper presented to the Society, April 6,1940, under the title On the number of representations of the square of an integer as the sum of an odd number of squares. The author wishes to thank Professor J. V. Uspensky for help in preparing this paper.

${ }^{2}$ The numbers in brackets refer to the bibliography. 